\title{
Dynamical gluon mass corrections in heavy quarkonia decays
}

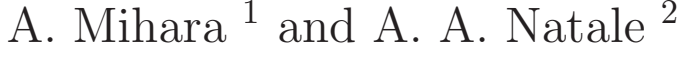 \\ Instituto de Física Teórica \\ Universidade Estadual Paulista \\ Rua Pamplona, 145 \\ 01405-900, São Paulo - SP \\ Brazil
}

\begin{abstract}
Using the expression of the dynamical gluon mass obtained through the operator product expansion we discuss the relevance of gluon mass effects in the decays $V \rightarrow$ hadrons $(V=J / \psi, \Upsilon)$. Relativistic and radiative corrections are also introduced to calculate $\alpha_{s}\left(m_{c}\right)$ and $\alpha_{s}\left(m_{b}\right)$ comparing them with other values available in the literature. The effects of dynamical gluon masses are negligible for $\Upsilon$ decay but important for $J / \psi$ decay.
\end{abstract}

PACS number(s): 13.20.Gd, 13.25.Gv, 14.40.Gx

\footnotetext{
${ }^{1}$ e-mail : mihara@ift.unesp.br

2e-mail : natale@ift.unesp.br
} 
Since the original suggestion by Appelquist and Politzer [1], the annihilation of heavy quark pairs into gluons or gluons plus a photon has been recognized as an apparently excellent process for testing the basic ideas of QCD and for measuring the strong coupling $\alpha_{s}$ with high precision.

One can determine $\alpha_{s}$ at low energies by comparing the experimental $\Upsilon$ and $J / \psi$ branching ratios

$$
R_{V}=\frac{\Gamma(V \rightarrow g g g)}{\Gamma(V \rightarrow e e)}, \quad V=\Upsilon, J / \psi .
$$

with the theoretical prediction. This is given, at the lowest order in perturbation theory, by

$$
R_{V}=\frac{10\left(\pi^{2}-9\right)}{81 \pi e_{q}^{2} \alpha\left(M_{V}\right)} \alpha_{s}^{3},
$$

where $e_{q}$ is the quark charge, $\alpha$ is the electromagnetic coupling and $M_{V}$ is the quarkonium mass. In addition, the experimental data for the radiative process $V \rightarrow \gamma+X$ is an important source of information about the QCD dynamics, which predicts a photon spectrum nearly linear in $z=2 E_{\gamma} / M_{V}$, where $E_{\gamma}$ is the photon energy.

The match of the experimental and theoretical determinations of $\alpha_{s}$ and the photon spectrum with high accuracy has originated a long series of discussions since the pioneer work of Ref. [1]. Corrections due to relativistic and radiative QCD effects were shown to modify Eq.(2). Other effects, as the existence of an effective gluon mass or beyond standard model contributions, are also possible explanations for the claimed differences between theory and experimental data. In this work we introduce the concept of a dynamical gluon mass and verify how it affects quarkonia decays. The fact that we consider a momentum-dependent gluon mass clearly modifies previous discussions on this problem.

Parisi and Petronzio [2] were the first to discuss some discrepancies between QCD results and the ones obtained from $J / \psi$ decays. They introduced an effective gluon mass to improve the comparison between experiment and theory in the case of $J / \psi$ decays. This is not the unique alternative used to solve the problems in this context but this line of thought does give reasonable results, and the possible effects of a gluon mass in quarkonia decays were considered several times in the literature (see [3]- [6] and references therein). In Refs. [2, 3, [1 the value of the gluon mass was determined calculating the CM energy spectrum of direct photons produced by $J / \psi$ and $\Upsilon$ decays $(J / \psi, \Upsilon \rightarrow g g \gamma)$. Afterwards the spectra were compared with experimental data, which clearly favoured the case of a massive gluon and fixed its 
mass. It is interesting to note that the explanation of the photon spectrum in the $J / \psi$ decay demanded a gluon mass $M_{g} \simeq 0.66 \mathrm{GeV}$, whereas in the $\Upsilon$ decay the most suitable mass value raised to $M_{g} \simeq 1.2 \mathrm{GeV}$ [3], 四]. There are some points that may be criticized in these results. With the knowledge accumulated in the past decades about the theory of the strong interactions it is obvious that we cannot have a bare gluon mass in QCD. In Refs. [3, [- the gluon masses differ in the $J / \psi$ and $\Upsilon$ decays in an unnatural way: as we increase the quarkonium mass the gluon mass needed to explain the photon spectrum is also increased. Finally, the explanation of the bending of the photon spectrum at large $z$ in these decays may be due to the radiation of additional gluons as described by Field [7].

It is clear that QCD does not admit a bare gluon mass. However, this theory may have a dynamical gluon mass [8] and recent simulations of QCD on the lattice strongly support this possibility [9]. Therefore, the proposal of Parisi and Petronzio [2] may indeed be realized in Nature, but in a more subtle way. This will be our working hypothesis. The next question is how to consider this dynamical mass in quarkonium decays. The introduction of a gluon mass scale in the calculation of some hadronic processes has been performed in a rather heuristic way (see, for instance, Ref. [10]). Only recently a more formal handling of gluon masses has been discussed [11]. In principle, we can justify the approach of Ref. [10] within the dynamical perturbation theory proposed by Pagels and Stokar many years ago [12, which basically imply in the use of the running gluon mass in the gluon propagator. This is the approach that we will follow here.

In order to obtain the asymptotic behavior of the gluon mass we can rely on the operator product expansion (OPE). This asymptotic behavior was obtained in Ref. [13]:

$$
M_{g}^{2}\left(P^{2}\right) \sim \frac{34 N \pi^{2}}{9\left(N^{2}-1\right)} \frac{\left\langle\frac{\alpha_{s}}{\pi} G^{\mu \nu} G_{\mu \nu}\right\rangle}{P^{2}},
$$

where $P^{2}\left(\equiv-p^{2}\right)$ is the gluon momentum in Euclidean space, $\left\langle\frac{\alpha_{s}}{\pi} G^{\mu \nu} G_{\mu \nu}\right\rangle$ is the gluon condensate [14, and $N=3$ for QCD. At this point we see the difference with previous work [3, 4]: the dynamical mass is connected with the gluon condensate and it decreases with energy. Therefore, we can expect different results from the ones of Refs. [3] and [4].

Equation (3) gives the asymptotic behavior of the gluon mass but we also need 
its expression in the infrared region. If we define

$$
m_{g}^{2} \equiv\left(\frac{34 N \pi^{2}}{9\left(N^{2}-1\right)}\left\langle\frac{\alpha_{s}}{\pi} G^{\mu \nu} G_{\mu \nu}\right\rangle\right)^{1 / 2}
$$

it was recently verified that the value of the dynamical gluon mass in the infrared is well described by the OPE value frozen at the scale $m_{g}$ [15], i.e. we can write

$$
M_{g}^{2}\left(P^{2}\right)=m_{g}^{2} \theta\left(m_{g}^{2}-P^{2}\right)+\frac{m_{g}^{4}}{P^{2}} \theta\left(P^{2}-m_{g}^{2}\right) .
$$

This is the expression which will be used in the calculation of quarkonium decays.

As discussed in Ref. [2], the gluon mass implies that the branching ratio $R_{V}$ must be changed to $R_{V} \cdot f_{3}(\eta)$, where

$$
f_{3}(\eta)=\frac{\left.\Gamma(V \rightarrow g g g)\right|_{m_{g}}}{\left.\Gamma(V \rightarrow g g g)\right|_{m_{g}=0}}
$$

is a function of $\eta \equiv 2 m_{g} / M_{V}$. This simple factorization is a consequence of the fact that most of the gluon mass contribution in the calculation of $\Gamma(V \rightarrow g g g)$ comes from the phase space integration [2, 5]. Therefore, we proceed in the same way as in [2] to compute $f_{3}$, with the difference that the mass is now a function of each final state gluon momentum.

In order to compute the function $f_{3}$ as a function of $\eta$ we made use of the package COMPHEP [16]. Initially we considered the case where a bare gluon mass was introduced in the matrix element and in the phase space. The result is given by the solid line in Fig.1, which is identical to the one of Ref. [2]. We then introduced a bare mass only in the phase space integration, obtaining the thin dashed line of Fig.1 (compatible with Ref.[3]). This result shows that the mass effect can be accounted for within this simple approximation. Once we verified that this approximation reproduces most of the mass effect, the calculation to obtain $f_{3}$ was performed by Monte Carlo integration considering the dynamical gluon mass contribution (Eq. (5)) only in the phase space. The result is the thick dashed line depicted in Fig.1.

As discussed before, in Refs. [2] and [3], the value of the gluon mass was obtained by calculating the CM energy spectrum of direct photons produced by $J / \psi$ and $\Upsilon$ decays $(J / \psi, \Upsilon \rightarrow g g \gamma)$, yielding very different values in each case. With the gluon mass obtained from the fit of the radiative quarkonia decays, the value of $f_{3}$ was determined from a curve similar to Fig.1, leading to a new determination of $\alpha_{s}$. Here the gluon mass $m_{g}$ is given by Eq. (4). If we consider the gluon condensate 


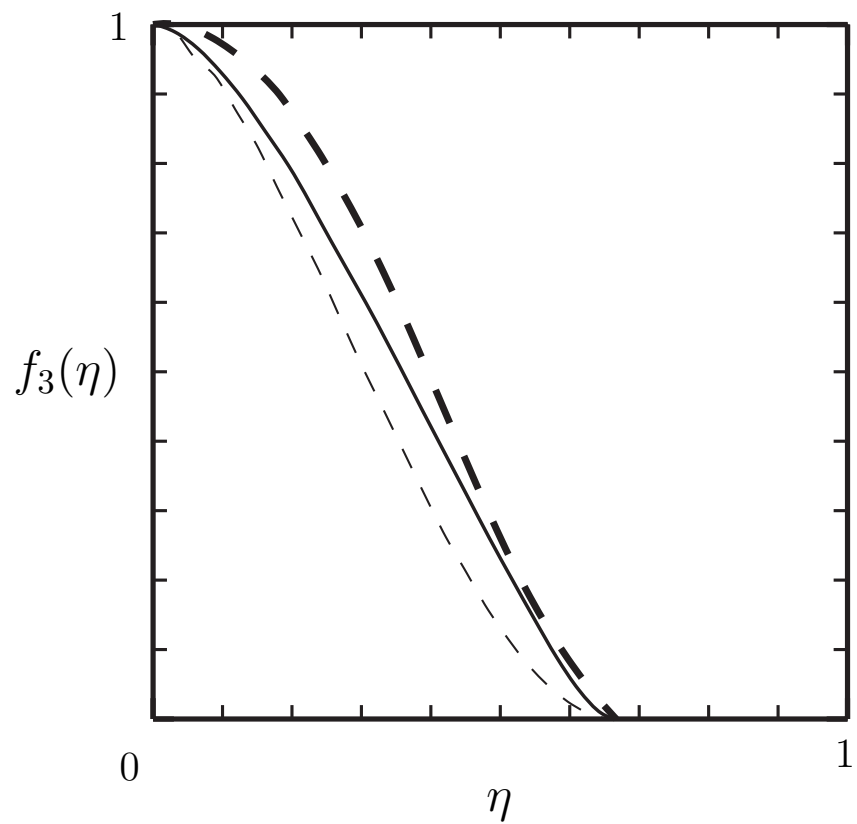

Figure 1: Function $f_{3}(\eta)$ representing the gluon mass corrections. The solid line represents these corrections when the effect of a bare gluon mass is taken into account both in the matrix element and phase space. The thin dashed line is obtained considering the effect of a bare gluon mass only in the phase space. The thick dashed line is our result obtained by taking into account a dynamical mass as given by Eq. (5).

value given in Ref. [14, 17], and the gluon mass obtained in Refs. [8, 10, 18], we can assume $m_{g} \approx(0.64 \pm 0.20) \mathrm{GeV}$, where the uncertainty is large enough to express the various limitations present in the dynamical gluon mass estimates. It is interesting to see that the central value is of the order of the value determined by Consoli and Field [3, [4] to explain the $J / \psi \rightarrow X+\gamma$ decay $\left(m_{g} \approx 0.66 \mathrm{GeV}\right)$. With this value for the gluon mass we certainly do not explain the direct photon spectrum in the inclusive decay of the $\Upsilon$. However, the explanation of this process may depend on a complex relationship between radiation of additional gluons as described by Field [7] and the existence of a dynamical gluon mass.

As one can notice in Fig.1, the new curve (the thick dashed one) is flatter than the curves of Refs. [2, 3] for small values of $\eta$. This is not so surprising since as $m_{g}$ decreases $f_{3}$ tends to 1 . But this feature is strengthened in the case of a dynamical mass whose momentum dependence is given by Eq.(5), because for small $\eta$ (which means small gluon mass or large quarkonium mass) the integrated phase space is larger than in the case of a bare gluon mass. This is so because we have 
configurations where at least one of the gluons resulting from the quarkonium decay has its mass effect sharply cut-off by a large momentum.

Using the values of $f_{3}$ from Fig.1 we can now calculate $\alpha_{s}\left(m_{c}\right)$ and $\alpha_{s}\left(m_{b}\right)$. Assuming $m_{g}=(0.64 \pm 0.20) \mathrm{GeV}$, we have $\left(\eta=2 m_{g} / M_{V}\right)$

$$
\eta^{(J / \psi)}=(0.41 \pm 0.13)
$$

and

$$
\eta^{(\Upsilon)}=(0.14 \pm 0.04)
$$

and from the curve of Fig.1, we obtain $f_{3}(\eta)$, i.e. the factors due to gluon mass corrections are

$$
f_{3}\left(\eta^{(J / \psi)}\right)=0.47 \pm 0.30, \quad f_{3}\left(\eta^{(\Upsilon)}\right)=0.94 \pm 0.03
$$

Our value of $f_{3}\left(\eta^{(J / \psi)}\right)$ is consistent with the one in Ref. [3, 4], but $f_{3}\left(\eta^{(\Upsilon)}\right)$ is approximately $30 \%$ larger and almost identical to 1 . If the mass was also introduced in the matrix element, the values of $f_{3}$ would be increased by a few percent and no signal of the gluon mass would appear for $f_{3}\left(\eta^{(\Upsilon)}\right)$.

We can now compute $\alpha_{s}$ from the $J / \psi$ and $\Upsilon$ decays, but to do so we have to take also into account the relativistic and QCD corrections. The QCD corrections are introduced through the factor (see [19])

$$
\left(1+\frac{\alpha_{s}}{\pi} b_{V}\right)
$$

where $b_{V}=1.6,0.43$ for $J / \psi$ and $\Upsilon$, respectively.

Equation (2) is obtained assuming that the $q \bar{q}$ pair annihilation occurs at a point. Relativistic corrections arise if we consider that this process occurs over a finite volume of radius $\simeq 1 / m_{q}$. Several papers have dealt with this issue. We follow Ref. [20] and use their correction factor: $\gamma^{(c)}=(0.31 \pm 0.03)$ for the $J / \psi$ and $\gamma^{(b)}=(0.69 \pm 0.07)$ for the $\Upsilon$.

Equation (21) rewritten with the correction factors mentioned above becomes

$$
R_{V}=f_{3}(\eta)\left(1+\frac{\alpha_{s}}{\pi} b_{V}\right) \gamma \frac{10\left(\pi^{2}-9\right)}{81 \pi e_{q}^{2} \alpha\left(M_{V}\right)} \alpha_{s}^{3}
$$

We then use the experimental values of $R_{V}$ from [21] and [4] to calculate $\alpha_{s}\left(m_{c}\right)$ and $\alpha_{s}\left(m_{b}\right): R_{J / \psi}=10.1 \pm 0.9$ and $R_{\Upsilon}=32.6 \pm 0.8$. Our results are

$$
\alpha_{s}\left(m_{c}\right)=0.35 \pm 0.07
$$


and

$$
\alpha_{s}\left(m_{b}\right)=0.206 \pm 0.008 \text {. }
$$

The values of Eqs.(12) and (13) are compatible with the ones of Refs. [4, 20. Note that Ref. [4] does not consider the relativistic corrections of Ref. [20]. On the other hand, our result for $f_{3}\left(\eta^{(\Upsilon)}\right)$ is about 1.6 times larger than the one of Refs. 3, [4. We find that the $\alpha_{s}\left(m_{b}\right)$ determination through $\Upsilon$ decays is barely affected by the dynamical gluon mass effects and, considering the relativistic corrections, it is totally consistent with the one of Ref.[3] (where the relativistic corrections were not considered). Contrary to the values of $\alpha_{s}\left(m_{c}\right)$ obtained in the references quoted above our result has a large error, and most of it, as we discuss next, is due to the uncertainty in the gluon mass.

Assuming that the gluon propagator has an infrared mass scale [9], we see several limitations in order to determine $\alpha_{s}$ with high confidence level through heavy quarkonium decays. The main problem of this approach is that the dynamical gluon mass scale is poorly known. The several determinations of this mass scale are characterized by a large range of possible values [8, 10, 18]. Even if we consider that this mass follows the behavior predicted by OPE [13], we know that the gluon condensate in opposition to the fermionic one, is known with a large uncertainty [17]. The extrapolation of this value to the infrared region also involves a series of approximations [15]. Finally, if we attempt to verify the consistency between measurements of $\alpha_{s}$ at different mass scales, we also may be in trouble if we do not take into account the gluon mass effect in the running coupling constant [22].

If the gluon propagator has a dynamical mass scale the strong running coupling constant is modified, at low energy, to [8, 22]

$$
\alpha_{s}\left(Q^{2}\right) \simeq \frac{12 \pi}{\left(11 N-2 n_{f}\right) \ln \left[\left(Q^{2}+\xi m_{g}^{2}\right) / \Lambda^{2}\right]},
$$

where $n_{f}$ is the number of fermion flavors, and $\xi \approx 4$ (or even larger [22]) is a parameter determined in Ref. [8]. This behavior of the running coupling constant with respect to the gluon mass appears when solving the Schwinger-Dyson equations for the gluon propagator and the trilinear vertex. The determination of Eq.(14) and its higher order corrections is a much more complex problem than the determination of the perturbative expression of $\alpha_{s}\left(Q^{2}\right)$, and, unfortunately, is not under complete control as the perturbative one. Therefore, at the scale $m_{b}$ and up we do not expect any substantial difference in the phenomenology if we use the perturbative running coupling constant when checking the consistency of $\alpha_{s}\left(m_{b}^{2}\right)$, obtained through Eq.(2), 
with others measurements of $\alpha_{s}$. At the scale $m_{c}$ this is not true anymore. In this case Eq.(14) and its higher order corrections (not yet computed) have to be used. At leading order of both coupling constants (perturbative and nonpertubative), the difference at the scale $m_{c}$ in using one or another expression amounts a factor roughly given by $\ln \left(m_{c}^{2}+\xi m_{g}^{2}\right) / \ln m_{c}^{2} \approx 1.6$. This number is large enough to interfere in high precision measurements of the running coupling constant using $J / \psi$ decays, and a better understanding of the gluon mass dependence of the running coupling constant is needed.

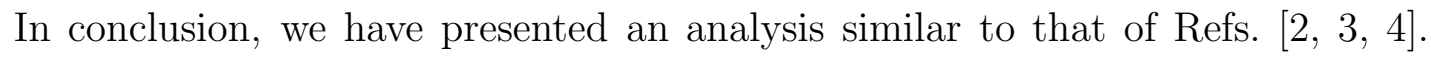
Notwithstanding, we considered the effects of a dynamical gluon mass, as given by Eq. (5), which is compatible with the asymptotic behavior predicted by OPE [13]. Monte Carlo integration of the phase space in quarkonium decays provided a new curve for the parameter $f_{3}(\eta)$, which is flatter for small values of $\eta$ than the previous ones, and reflects the momentum dependence of the dynamical gluon mass. Assuming $m_{g} \approx(0.64 \pm 0.20) \mathrm{GeV}$, we have obtained new values for $f_{3}\left(\eta^{J / \psi}\right)$ and $f_{3}\left(\eta^{\Upsilon}\right)$. With these values we calculated $\alpha_{s}\left(m_{c}\right)$ and $\alpha_{s}\left(m_{b}\right)$ including QCD as well as relativistic corrections. The values of $\alpha_{s}$ which we have obtained, within the assumed error bars for the gluon masses, are compatible with the ones in Refs. [- 4 , 20]. Contrarily to previous analysis we verified that $\alpha_{s}\left(m_{b}\right)$ is not affected by the existence of a dynamical gluon mass. More rigorous determinations of the gluon mass scale are necessary in order to determine $\alpha_{s}\left(m_{c}\right)$ with high precision by using heavy quarkonium decays.

\section{Acknowledgments}

We are grateful to R. Rosenfeld for useful discussions. This research was supported in part by the Conselho Nacional de Desenvolvimento Científico e Tecnológico (CNPq) (AAN), Coordenadoria de Aperfeiçoamento de Pessoal de Ensino Superior (CAPES) (AM), Fundação de Amparo à Pesquisa do Estado de São Paulo (FAPESP) (AAN), and by Programa de Apoio a Núcleos de Excelência (PRONEX). 


\section{References}

[1] T. Appelquist and H. D. Politzer, Phys. Rev. D12 (1975) 1404.

[2] G. Parisi and R. Petronzio, Phys. Lett. 94B (1980) 51 .

[3] M. Consoli and J. H. Field, Phys. Rev. D49 (1994) 1293 ; J. H. Field, "Perturbative QCD phenomenology with massive gluons", University of Geneva Report No. UGVA-DPNC 1993/2-155 (1993) (unpublished); idem, Int. J. Mod. Phys. A9 (1994) 3283 .

[4] M. Consoli and J. H. Field, J. Phys. G23 (1997) 41.

[5] M. L. Mangano and A. Petrelli, Phys. Lett. B351 (1995) 445 .

[6] M. Anselmino and F. Murgia, Phys. Rev. D53 (1996) 5314 ; F. Murgia, Phys. Rev. D54 (1996) 3365 .

[7] R. D. Field, Phys. Lett. 133B (1983) 248 .

[8] J. M. Cornwall, Phys. Rev. D26 (1982) 1453.

[9] F. D. R. Bonnet, P. O. Bowman, D. B. Leinweber and A. G. Williams, ADP00-10/T396 (hep-lat/0002020); D. B. Leinweber et al. (UKQCD Collaboration) Phys. Rev. D60, 094507 (1999); Phys. Rev. D58 (1998) 031501 ; C. Bernard, C. Parrinello, and A. Soni, Phys. Rev. D49 (1994) 1585 ; P. Marenzoni, G. Martinelli, N. Stella, and M. Testa, Phys. Lett. B318 (1993) 511 ; P. Marenzoni et al., Published in Como Quark Confinement (1994) pp. 210-212 (QCD162:Q83:1994); R. Gupta et al., Phys. Rev. D36 (1987) 2813 ; J. E. Mandula and M. Ogilvie, Phys. Lett. B185 (1987) 127 ; P. A. Amundsen and J. Greensite, Phys. Lett. B173 (1986) 179 , C. Bernard, Phys. Lett. B108 (1982) 431.

[10] F. Halzen, G. Krein, and A. A. Natale, Phys. Rev. D47 (1993) 295 ; M. B. Gay Ducati, F. Halzen, and A. A. Natale, Phys. Rev. D48 (1993) 2324 ; J. R. Cudell and B. U. Nguyen, Nucl. Phys. B420 (1994) 669 .

[11] J. R. Forshaw, J. Papavassiliou and C. Parrinello, Phys. Rev. D59 (1999) 074008 .

[12] H. Pagels and S.Stokar, Phys. Rev. D20 (1979) 2947. 
[13] M. Lavelle, Phys. Rev. D44 (1991) R26 .

[14] M. A. Shifman, A. I. Vainshtein, M. B. Voloshin and V. I. Zakharov, Phys. Lett. B77 (1978) 80 ; A. Vainshtein, V. Zakharov and M. Shifman, JETP Lett. 27 (1978) 55 ; M. A. Shifman, A. I. Vainshtein and V. I. Zakharov, Nucl. Phys. B147 (1979) 385, 448 .

[15] E. V. Gorbar and A. A. Natale, Phys. Rev. D61 (2000) 054012.

[16] E. E. Boos, M. N. Dubinin, V. A. Ilyin, A. E. Pukhov and V. I. Savrin, COMPHEP: Specialized Package for Automatic Calculations of Elementary Particle Decays and Collisions, SNUTP-94-116 (hep-ph/9503280).

[17] S. Narison, Nucl. Phys. Proc. Suppl. 54A (1997) 238.

[18] J. C. Montero, A. A. Natale and P. S. Rodrigues da Silva, Phys. Lett. B406 (1997) 130 .

[19] W. Kwong et al. , Phys. Rev. D37 (1988) 3210 .

[20] H. C. Chiang, J. Hüfner and H. J. Pirner, Phys. Lett. B 324 (1994) 482 .

[21] Particle Data Group, Phys. Rev. D45 (1992) 1.

[22] J. M. Cornwall and J. Papavassiliou, Phys. Rev. D40 (1989) 3474 ; J. Papavassiliou and J. M. Cornwall, Phys. Rev. D44 (1991) 1285. 